\title{
Assessing the Prevalence and Predicting Factors of an Abnormal Gated Myocardial Perfusion SPECT in Asymptomatic Patients with Type 2 Diabetes
}

\author{
Elinaz Hosseinzadeh ${ }^{1}$, Mohammad ALi Ghodsirad ${ }^{2}$, Toktam Alirezaei ${ }^{1}$, MAryam Arefnia ${ }^{1}$, \\ Mahasti Amoui $^{1}$, Elaheh Pirayesh ${ }^{1}$, Ghazal Norouzi ${ }^{1}$, and Sepideh Khoshbakht ${ }^{1}$ \\ ${ }^{1}$ Affiliation not available \\ ${ }^{2}$ Shaheed Beheshti University of Medical Sciences
}

May 11, 2021

\begin{abstract}
Abstract Introduction: Considering the significant prevalence of silent myocardial ischemia and its related morbidity and mortality in asymptomatic type two diabetic patients, it is not well known whether early screening with MPI is cost-effective. However, predicting factors are not elucidated. Materials and Methods: This was a cross-sectional study including 63 asymptomatic patients with type 2 diabetes mellitus (T2DM), with normal ECG and ejection fraction. Patients with any history of documented valvular, congestive or ischemic heart disease, renal or hepatic failure were excluded. At first all patients were interviewed and checked for risk factors and then patients underwent a two-day rest/stress 99mTc-MIBI gated MPI SPECT. Data was assessed by QPS/QGS and 4DM software and evaluated by a nuclear medicine specialist with summed stress score (SSS) of more than 4 defined as CAD. Results: There were 42 females (67\%) and 21 males (33\%), with a mean age of $61.33 \pm 6.98$ years and $7.97 \pm 4.86$ years history of T2DM. CAD was detected in $26(41.3 \%)$ patients and was significantly associated with male gender, smoking, requiring insulin therapy and $\mathrm{EF}$ ( $\mathrm{P}$-value $=0.019,0.046,0.05,0.033$, respectively). A significant association was found between the duration of diabetes, especially when $>15 y$, and the probability of having CAD.. Multivariable logistic regression revealed that smoking; male gender and diabetes duration were the strongest independent predictors of abnormal MPI results. Conclusion: We found a high (46\%) prevalence of abnormal stress MPI SPECT in patients with type 2 diabetes mellitus, despite being asymptomatic. Asymptomatic patients with a history of smoking, long duration of diabetes, being under insulin treatment and male gender might benefit from MPI for early detection of silent ischemia. Keyword: 99mTc-MIBI; Gated Myocardial Perfusion Imaging; Type 2 Diabetes Mellitus; Silent Ischemia; Asymptomatic CAD; Early Screening
\end{abstract}

\begin{abstract}
Introduction: Considering the significant prevalence of silent myocardial ischemia and its related morbidity and mortality in asymptomatic type two diabetic patients, it is not well known whether early screening with MPI is cost-effective. However, predicting factors are not elucidated.

Materials and Methods: This was a cross-sectional study including 63 asymptomatic patients with type 2 diabetes mellitus (T2DM), with normal ECG and ejection fraction. Patients with any history of documented valvular, congestive or ischemic heart disease, renal or hepatic failure were excluded. At first all patients were interviewed and checked for risk factors and then patients underwent a two-day rest/stress 99mTc-MIBI gated MPI SPECT. Data was assessed by QPS/QGS and 4DM software and evaluated by a nuclear medicine specialist with summed stress score (SSS) of more than 4 defined as CAD.
\end{abstract}

Results: There were 42 females $(67 \%)$ and 21 males (33\%), with a mean age of $61.33 \pm 6.98$ years and $7.97 \pm$ 4.86 years history of T2DM. CAD was detected in $26(41.3 \%)$ patients and was significantly associated with 
male gender, smoking, requiring insulin therapy and $\mathrm{EF}$ (P-value $=0.019,0.046,0.05,0.033$, respectively). A significant association was found between the duration of diabetes, especially when $>15 \mathrm{y}$, and the probability of having CAD.. Multivariable logistic regression revealed that smoking; male gender and diabetes duration were the strongest independent predictors of abnormal MPI results.

Conclusion: We found a high (46\%) prevalence of abnormal stress MPI SPECT in patients with type 2 diabetes mellitus, despite being asymptomatic. Asymptomatic patients with a history of smoking, long duration of diabetes, being under insulin treatment and male gender might benefit from MPI for early detection of silent ischemia.

Keyword: 99mTc-MIBI; Gated Myocardial Perfusion Imaging; Type 2 Diabetes Mellitus; Silent Ischemia; Asymptomatic CAD; Early Screening

Highlights:

- The prevalence of type2 DM is increasing

- Many patients with type 2 diabetes develop CAD

- The optimal CAD screening tool for diabetics is unknown

- Myocardial perfusion study (MPS) is a non-invasive and effective diagnostic method for CAD

- Which asymptomatic diabetics would benefit the most form MPS is controversial.

- Type 2 diabetics with longer duration of diabetes, smokers and insulin users are at greater risk of asymptomatic CAD

\section{Introduction}

The global prevalence of type 2 diabetes mellitus (T2 DM), an essential risk factor for coronary artery disease $(\mathrm{CAD})$, is on the rise $(1,2)$. On the other hand, CAD as a major cause of morbidity, mortality, and financial costs in patients with T2DM, could be asymptomatic in 20-35\% of diabetic patients (3). However, acute myocardial infarction (MI) and sudden cardiac death might be often the initial presentations $(4,5)$. Developing at a younger age, higher rate of multi-vessel disease and being more prone to have complications after MI, such as congestive heart failure, are some of the features indicating the overall poorer outcome of CAD in diabetic patients (6-8). This further emphasizes the importance of detecting CAD at an early stage in these patients (9). It has been suggested that myocardial perfusion imaging (MPI) is a reliable technique for risk assessment of CAD in diabetic patients (10-12). It is still under debate whether screening myocardial ischemia is rational in asymptomatic diabetic patients compared to standard therapeutic strategies and whether it improves the risk stratification, management, and prognosis in different patient groups (4). Therefore, the aim of this study was to assess CAD risk, prevalence and Predicting Factors of an Abnormal Gated Myocardial Perfusion SPECT in Asymptomatic Patients with Type 2 Diabetes .

\section{Materials and Methods}

This was a cross-sectional study, conducted at the nuclear medicine department of Shohada Tajrish hospital, Tehran, Iran. In total, 63 asymptomatic patients with type 2 DM, referred for check-up or pre-operative cardiac evaluation entered the study. An ethical approval was obtained from Shahid Beheshti Medical University Ethics committee.

Patients were interviewed on the appointed scanning day and informed about the goals of study in detail. A written informed consent was obtained from all patients before enrolment. The inclusion criteria were patients with proven T2DM, aged 35-80 years, ejection fraction $(\mathrm{EF})>50 \%$, normal ECG and life expectancy of more than one year. Patients with any history of valvular heart disease, ischemic heart disease, abnormal renal or hepatic failure, coronary intervention, arrhythmia, perfusion defect on prior MPI scans or wall motion abnormality on prior gated stress SPECT-MPI were excluded. Demographic characteristics of participants were documented in a questionnaire. All patients underwent a two-day rest/stress 99mTc-MIBI gated MPI SPECT, according to the European Association of Nuclear Medicine (EANM) guidelines for MPI scan 
(13). The rest scan was performed 60 minutes after IV injection of $555 \mathrm{MBq} 99 \mathrm{mTc}$-sestamibi. On the day after, myocardial stress was achieved by exercise (Bruce protocol) or Dipyridamole $(0.14 \mathrm{mg} / \mathrm{kg} / \mathrm{min}$ over 4 minutes), with $740 \mathrm{MBq} 99 \mathrm{mTc}-$ sestamibi injected at peak stress. All patients consumed $120 \mathrm{~mL}$ whole milk 10 minutes after tracer injection, as per routine. Imaging was performed in both supine and prone positions. SPECT images were acquired with a variable angle dual head Evo-Voxel Siemens gamma camera (64 projections; 25 seconds/projection, matrix $64 * 64$, zoom of 1.46, 8-frame gated study, low-energy high resolution collimator, in a noncircular orbit). The images were processed using QPS/QGS and 4DM software and the results were interpreted by a nuclear medicine specialist.

\section{Statistical Analysis}

Descriptive data were reported as mean and standard deviation for quantitative variables and number and percentage for qualitative variables. T-test was used to compare the means between the two groups. For all tests, a P-value below 0.05 was considered statistically significant. SPSS version 20 (SPSS Inc., Chicago, IL, The USA) was used for data analysis.

\section{Results}

Of 63 patients, 42 were females $(67 \%)$ and 21 males (33\%), with a mean age of $61.33 \pm 6.98$ years (range: 42-78). The mean \pm SD duration of diabetes was $7.97 \pm 4.86$ years. Baseline characteristics of participants are presented in Tables $1 \& 2.54$ patients underwent stress with Dipyridamole, 2 with Dobutamine and 7 with exercise.

According to the obtained values of MPI (true SSS > 4, without attenuation artifacts), CAD was detected in $26(41.3 \%)$ patients. Presence of CAD was significantly associated with male gender, smoking and EF (P-values; 0.019, 0.046, 0.033, respectively). While hypertension and dyslipidemia were more common in CAD patients compared to those without CAD, no statistically significant association was noted (P-value $>$ 0.05) (Table 3).

Moreover, patients were divided into five groups based on the duration of diabetes. Kruskal Wallis analysis revealed a significant correlation between the duration of diabetes, especially when more than 15 years, and the probability of CAD, based on SSS, SDS especially in LAD territory (P-value $=0.048$ ) (Figures 1 ). Besides, requiring Insulin therapy was significantly more prevalent in diabetic patients with CAD compared to those without CAD $(\mathrm{P}$-value $=0.042)$. $($ Figure 2$)$

Mean EDV and ESV were higher in CAD patients compared to diabetic patients without CAD (68.52 \pm 3.41 and $29.35 \pm 2.14 \mathrm{~mL}$ versus $52.72 \pm 2.23$ and $18.52 \pm 1.47 \mathrm{~mL})$. However, the difference was not statistically significant $(\mathrm{P}$-value $>0.05)$.

According to multivariate logistic analysis, smoking had a significant association with SDS, RCA territory involvement and RV visualization. In addition, duration of DM was significantly associated with higher SSS and SRS, presence of TID, and RCA/LAD territory involvement. Smoking, male gender and diabetes duration were the strongest independent predictors of abnormal MPI results.

Univariant linear regression analysis showed an association between SSS, SRS, LAD/RCA territories involvement and TID with the duration of diabetes. SRS, RCA territory involvement and EF were also independently associated with hyperlipidemia. LAD territory involvement was associated with insulin therapy, HTN, and RV visualization. TID was associated with neuropathy and peripheral arterial disease (PAD). SDS was associated with B-blocker therapy.

Table 1. Demographic characteristics of the study participants

\begin{tabular}{ll}
\hline Male/female & $21(33.33 \%) / 42(66.67 \%)$ \\
\hline Age(years) & 61 \\
Diabetes duration(years) & 7.97 \\
Treatment & Treatment
\end{tabular}




\begin{tabular}{ll}
\hline Male/female & $21(33.33 \%) / 42(66.67 \%)$ \\
\hline Insulin & $14(22.22 \%)$ \\
Insulin+oral agent & $10(15.87 \%)$ \\
Oral agent & $39(61.90 \%)$ \\
Retinopathy(yes/no) & $10(16.13 \%) / 52(83.87 \%)$ \\
Peripheral vascular disease & $6(9.68 \%) / 56(90.32 \%)$ \\
ASA & $24(42.11 \%)$ \\
HTN (yes/no) & $35(55.56 \%) / 28(44.44 \%)$ \\
HLP (yes/no) & $36(57.14 \%) / 27(42.86 \%)$ \\
Mean TG & 121 \\
Mean Cholesterol & 169 \\
Mean HDL mg/dl & 41 \\
Mean LDL mg/dl & 94 \\
Mean BMI & 28.60 \\
HbA1c\% & 7.1 \\
Smoking (yes/no) & $10(5.87 \%) / 53(84.13 \%)$ \\
Neuropathy (yes/no) & $5(8.20 \%) / 56(91.8 \%)$ \\
Nephropathy (yes/no) & $7(11.48 \%) / 54(88.52 \%)$ \\
Family history (yes/no) & $9(14.52 \%) 53(85.48 \%)$ \\
b-blocker & $25(40.98 \%)$ \\
Statin & $39(63.93 \%)$ \\
ACE inhibitor & $33(56.90 \%)$ \\
\hline
\end{tabular}

*Based on Chi-Square Test; HTN= hypertension; HLP = high lipid profit; DIP = dipyridamole. TG: Triglyceride,

HDL: High density lipoprotein, LDL: Low density lipoprotein, BMI: Body mass index, ACE : Angiotensinconverting enzyme

Table 2. MPI SCAN Results

\begin{tabular}{ll}
\hline Index & Mean \pm SD \\
\hline EDV & $59.54 \pm 2.87$ \\
ESV & $22.44 \pm 1.65$ \\
EF & $56.93 \pm 8.84$ \\
SDS & $2.51 \pm 3.53$ \\
SRS & $1.38 \pm 2.84$ \\
SSS & $4.00 \pm 4.35$ \\
Dilated LV & $5(7.94 \%)$ \\
RV & $4(6.35 \%)$ \\
TID & $6(9.52 \%)$ \\
LCX & $8(12.70 \%)$ \\
LAD & $23(36.51 \%)$ \\
RCA & $11(17.46 \%)$ \\
\hline
\end{tabular}

EDV: End diastolic value, ESV: End systolic value, EF: Ejection fraction,

SDS: Summed difference score, SRS: Summed rest score, SSS: Summed stress score, 
LV: left ventricle, RV: Right ventricle, TID: Transient ischemic dilatation,

LCX: Left circumflex artery, LAD: Left ascending artery, RCA: Right coronary artery

Table 3. Comparing Patients with Normal and abnormal MPI Scan

\begin{tabular}{llll}
\hline Variable & Non-CAD & CAD & P value \\
\hline N & $37(58.7 \%)$ & $26(41.3 \%)$ & \\
Age & $59.35 \pm 6.26$ & $64.15 \pm 7.09$ & 0.301 \\
Male/female & $8(21.6 \%) / 29(78.4 \%)$ & $13(50 \%) / 13(50 \%)$ & 0.019 \\
HbA1c & $7.73 \pm 2.16$ & $7.11 \pm 1.23$ & 0.155 \\
HTN & $18(48.6 \%)$ & $17(65.4 \%)$ & 0.118 \\
HLP & $20(54.1 \%)$ & $16(61.5 \%)$ & 0.371 \\
SMOKING & $4(26.9 \%)$ & $7(10.8 \%)$ & 0.094 \\
Neuropathy & $2(5.6 \%)$ & $3(12 \%)$ & 0.329 \\
Retinopathy & $6(16.7 \%)$ & $4(15.4 \%)$ & .584 \\
Nephropathy & $6(16.7 \%)$ & $1(4 \%)$ & 0.131 \\
PAD & $2(5.6 \%)$ & $4(15.4 \%)$ & 0.196 \\
FH & $6(16.7 \%)$ & $3(11.5 \%)$ & .427 \\
B-blocker & $11(31.4 \%)$ & $17(53.8 \%)$ & .078 \\
ASA & $13(46 \%)$ & $11(44 \%)$ & .798 \\
ACEI & $17(53.1 \%)$ & $16(63.5 \%)$ & .354 \\
Statins & $21(60.0 \%)$ & $18(69.2 \%)$ & .320 \\
BMI & $28.08 \pm 5.99$ & $28.3 \pm 4.13$ & .698 \\
EF & $67.91 \pm 9.29$ & $63.11 \pm 8.54$ & .033 \\
EDV & $52.72 \pm 3.41$ & $68.52 \pm 2.14$ & .097 \\
ESV & $18.53 \pm 2.23$ & $29.35 \pm 1.47$ & .089 \\
LV dilated & 0 & $5(19.2 \%)$ & \\
RV & 0 & $4(15.4 \%)$ & \\
TID & 0 & $6(23.1 \%)$ & \\
\hline
\end{tabular}

HTN; Hypertension, HLP; Hyperlipidemia, PAD; Peripheral artery disease, FH; Family History, ASA; Aspirin, ACEI; Angiotensin-converting enzyme inhibitors, BMI; Body Mass Index, EF; Ejection fraction, EDV; End-diastolic volume, ESV; End-systolic volume, RV; Right ventricle, TID; Transient ischemic dilatation.

Figure 1. Duration of Diabetes in Patients With and Without CAD. Numbers in the vertical axis indicate number of patients. Color bars correspond to the duration of diabetes in year. CAD; coronary artery disease. 


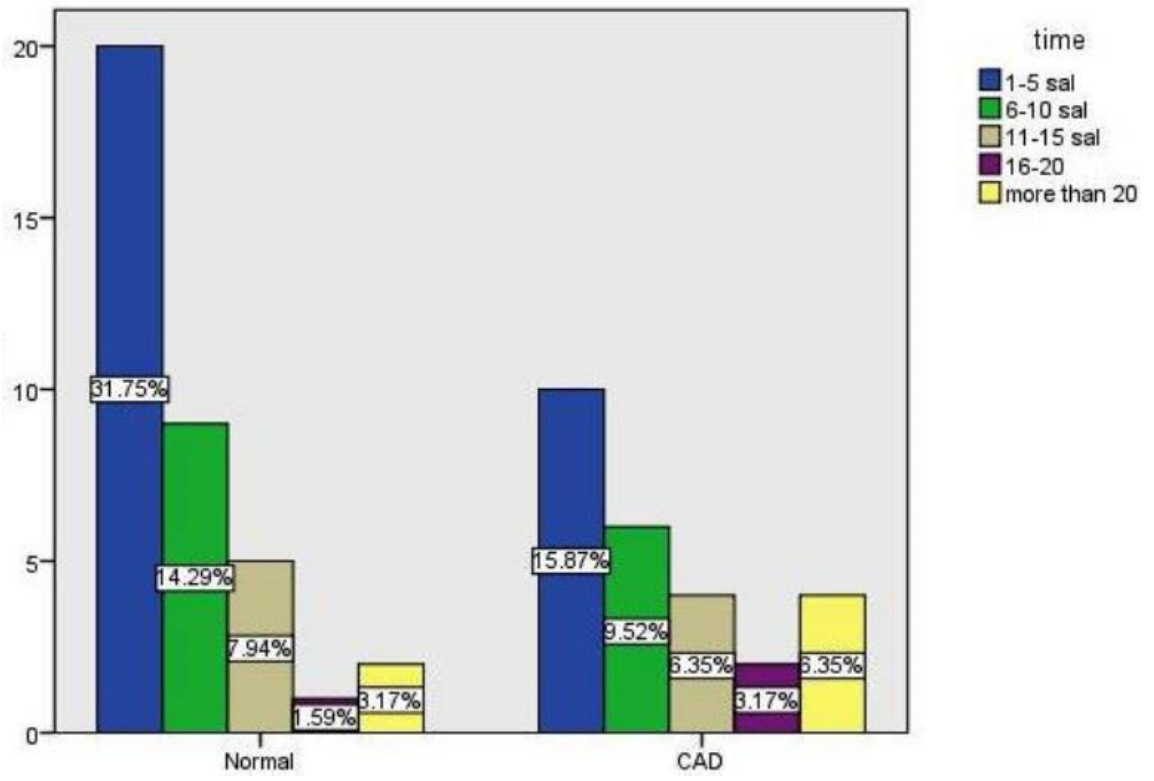

Figure 2. Type of Treatment in Patients With and Without CAD. Numbers in the vertical axis indicate number of patients.

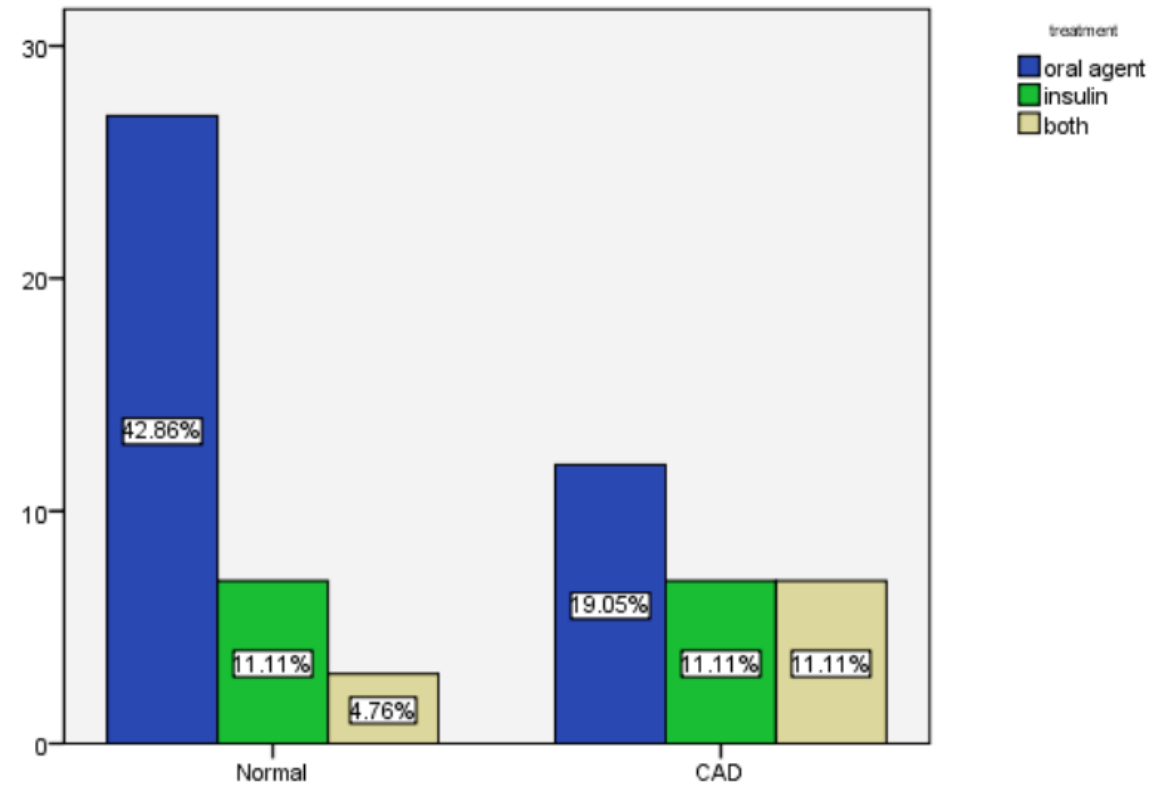

\section{Discussion}

Many studies have been conducted to evaluate the prevalence of silent myocardial ischemia in asymptomatic diabetic patients, reporting a prevalence of $9 \%$ to $57 \%$ (14). Silent ischemia is characterized by confirmed myocardial ischemia objectively without symptoms of angina or angina equivalents(15). The autonomic neuropathy in diabetes has been proposed to be the reason for blunted perception of chest pains, justifying a 
higher proportion of silent ischemia in these patients (16). The incidence of cardiovascular events and death are significantly higher in type 2 diabetic patients with MPI SPECT abnormalities (17).

Various diagnostic tests are implemented to diagnose CAD, each having their own limitations. For example, rest ECG could yield normal findings in more than a half of patients with chronic stable angina (18). Also, no agreement has been achieved on which asymptomatic diabetic patients should be screened $(19,20)$. Scholte et al. reported a $13 \%$ rate of cardiac deaths and cardiovascular events in patients with type 2 diabetes during a 3-year follow-up. None of the known cardiovascular risk factors have been shown to have enough sensitivity and specificity to predict silent myocardial ischemia in diabetic patients (21).

However, our study revealed a $41.3 \%$ prevalence of silent ischemia in asymptomatic type 2 diabetic patients, higher than $37 \%$ reported by Al-Humaidi et al (22). They reported that perfusion defects were related to disease duration, insulin use, nephropathy, and neuropathy. Their results regarding the duration of diabetes or insulin use was in accordance with our findings which should be examined in further studies as well.

CAD was diagnosed in our patients despite having no compromise in LVEF in echocardiography or electrocardiographic (ECG), highlighting the superior sensitivity of MPI SPECT in detecting silent ischemia/CAD. This is in accordance with the findings of Mohagheghie et al. who reported that CAD was detected by MPI in one third of asymptomatic diabetic patients with normal ECG and no evidence of peripheral arterial disease (23). Moreover, CAD was significantly more prevalent in patients with diabetes mellitus of more than 15 years, consistent with other studies which indicate an increased risk of cardiac events in prolonged diabetes mellitus (24-26).

$\mathrm{Wu}$ et al. reported that asymptomatic postmenopausal women with DM had a higher SSS and SDS than age-matched men (27). While our study found that male gender, regardless of smoking and requiring insulin treatment, is a strong predictor of myocardial perfusion abnormalities in asymptomatic diabetic patients. This might be due to poor control of blood sugar in men and intentional non-adherence to medications compared to women. However, women in our study were mainly pre-menopausal.

Moreover, Salehi et al, concluded that DM was independently associated with abnormal MPI in asymptomatic patients with a normal LVEF, also claiming that smoking and longer duration of DM were correlated with myocardial perfusion abnormalities, which is consistent with our findings. However, they found no significant difference between males and females in terms of abnormal MPI findings (28). This might be due to different study population or inclusion criteria.

MPI SPECT is a readily accessible, easily performed modality compared to other evolving modalities such as CT-angiography, MRI or newly discovered biomarkers, as evidence is scarce in supporting their incremental values in predicting further cardiac events. Moreover, MPI SPECT offers an automated evaluation and is less operator-dependent (29).

Our study had some limitations. Our sample size was quite small. Besides, the effect of some of possible confounding factors such as underlying liver or kidney disease (abnormal liver function tests or serum creatinine levels) was not assessed in our study, as we excluded such patients from our study. Further, prospective studies, with larger population and longer follow-ups are necessary to determine the prognostic value of an abnormal MPI in asymptomatic diabetic patients.

\section{Conclusion}

The current study revealed a high (46\%) prevalence of abnormal stress MPI SPECT in patients with type 2 diabetes mellitus, despite having a normal ECG and EF in echocardiography, which in turn indicates a higher risk of fatal cardiac events, if not detected early. Smoking, duration of diabetes, requiring insulin treatment and male gender were independent predictors of abnormal MPI, proceeding to silent ischemia. These findings may assist cardiologists in choosing asymptomatic diabetic patients for early screening of $\mathrm{CAD}$, thereby lowering the risk of CAD-related morbidity and mortality.

\section{Acknowledgment}


We would like to thank the staff of nuclear medicine department of Shohada-e Tajrish hospital for helping in collecting relevant data.

\section{Funding and support}

None

\section{Financial disclosure}

None

\section{Authors' contribution}

All the authors participated in preparing the manuscript according to the ICMJE authorship criteria.

\section{References}

1. Boiten HJ, van Domburg RT, Valkema R, Zijlstra F, Schinkel AF. Dobutamine stress myocardial perfusion imaging: 8-year outcomes in patients with diabetes mellitus. European Heart Journal-Cardiovascular Imaging. 2016;17(8):871-6.

2. Zellweger MJ, Haaf P, Maraun M, Osterhues HH, Keller U, Müller-Brand J, et al. Predictors and prognostic impact of silent coronary artery disease in asymptomatic high-risk patients with diabetes mellitus. International journal of cardiology. 2017;244:37-42.

3. Weinrauch L, D'elia J, Healy R, Gleason R, Takacs F, Libertino J, et al. Asymptomatic coronary artery disease: angiography in diabetic patients before renal transplantation: relation of findings to postoperative survival. Annals of internal medicine. 1978;88(3):346-8.

4. Mitevska IP, Baneva N, Srbinovska E, Stojanovska L, Apostolopoulos V, Bosevski M. Prognostic implications of myocardial perfusion imaging and coronary calcium score in a Macedonian cohort of asymptomatic patients with type 2 diabetes. Diabetes and Vascular Disease Research. 2017;14(4):285-94.

5. Fishman SL, Sonmez H, Basman C, Singh V, Poretsky L. The role of advanced glycation end-products in the development of coronary artery disease in patients with and without diabetes mellitus: a review. Molecular Medicine. 2018;24(1):1-12.

6. Naito R, Miyauchi K. Coronary artery disease and type 2 diabetes mellitus current treatment strategies and future perspective. International heart journal. 2017:17-191.

7. Sanchez-Alcoholado L, Castellano-Castillo D, Jordán-Martínez L, Moreno-Indias I, Cardila-Cruz P, Elena $\mathrm{D}$, et al. Role of gut microbiota on cardio-metabolic parameters and immunity in coronary artery disease patients with and without type-2 diabetes mellitus. Frontiers in microbiology. 2017;8:1936.

8. Kim T-H, Yang P-S, Uhm J-S, Kim J-Y, Pak H-N, Lee M-H, et al. CHA2DS2-VASc score (congestive heart failure, hypertension, age[?] 75 [doubled], diabetes mellitus, prior stroke or transient ischemic attack [doubled], vascular disease, age 65-74, female) for stroke in Asian patients with atrial fibrillation: a Korean nationwide sample cohort study. Stroke. 2017;48(6):1524-30.

9. Wiersma JJ, Verberne HJ, Trip MD, Wik L, van Eck-Smit BL, Piek JJ, et al. Prevalence of myocardial ischaemia as assessed with myocardial perfusion scintigraphy in patients with diabetes mellitus type 2 and mild anginal symptoms. European journal of nuclear medicine and molecular imaging. 2006;33(12):1468-76.

10. Morales DCV, Bhavnani SP, Ahlberg AW, Pullatt RC, Katten DM, Polk DM, et al. Coronary risk equivalence of diabetes assessed by SPECT-MPI. Journal of Nuclear Cardiology. 2019;26(4):1093-102.

11. Colon CM, Marshell RL, Roth CP, Farag AA, Iskandrian AE, Hage FG. The prognostic value of myocardial perfusion imaging in patients with type 2 myocardial infarction. Journal of Nuclear Cardiology. 2019:1-10. 
12. Acampa W, Assante R, Zampella E, Petretta M, Cuocolo A. Myocardial perfusion imaging for diabetes: Key points from the evidence and clinical questions to be answered. Journal of Nuclear Cardiology. 2019:1-9.

13. Gimelli A, Achenbach S, Buechel RR, Edvardsen T, Francone M, Gaemperli O, et al. Strategies for radiation dose reduction in nuclear cardiology and cardiac computed tomography imaging: a report from the European Association of Cardiovascular Imaging (EACVI), the Cardiovascular Committee of European Association of Nuclear Medicine (EANM), and the European Society of Cardiovascular Radiology (ESCR). European heart journal. 2018;39(4):286-96.

14. Oki GCR, Pavin EJ, Coelho OR, Parisi MCR, Almeida RC, Etchebehere ECSdC, et al. Myocardial perfusion scintigraphy in the detection of silent ischemia in asymptomatic diabetic patients. Radiologia Brasileira. 2013;46(1):7-14.

15. Cohn PF, Fox KM, of WtA, Daly C. Silent myocardial ischemia. Circulation. 2003;108(10):1263-77.

16. Prior JO, Monbaron D, Koehli M, Calcagni M-L, Ruiz J, Delaloye AB. Prevalence of symptomatic and silent stress-induced perfusion defects in diabetic patients with suspected coronary artery disease referred for myocardial perfusion scintigraphy. European journal of nuclear medicine and molecular imaging. 2005;32(1):60-9.

17. Yamasaki Y, Nakajima K, Kusuoka H, Izumi T, Kashiwagi A, Kawamori R, et al. Prognostic value of gated myocardial perfusion imaging for asymptomatic patients with type 2 diabetes: the J-ACCESS 2 investigation. Diabetes Care. 2010;33(11):2320-6.

18. Scholte AJ, Schuijf JD, Kharagjitsingh AV, Dibbets-Schneider P, Stokkel MP, van der Wall EE, et al. Prevalence and predictors of an abnormal stress myocardial perfusion study in asymptomatic patients with type 2 diabetes mellitus. European journal of nuclear medicine and molecular imaging. 2009;36(4):567-75.

19. Kawasaki-Ogita Y, Hamamoto Y, Honjo S, Ikeda H, Wada Y, Nomura K, et al. The limited usefulness of the treadmill test or a risk-guided approach in screening for asymptomatic coronary heart disease in Japanese patients with type 2 diabetes. Internal Medicine. 2012;51(24):3337-42.

20. Prior JO. Myocardial Perfusion Scintigraphy in Diabetes: Current Status and Limitations. Integrating Cardiology for Nuclear Medicine Physicians: Springer; 2009. p. 305-23.

21. Cosson E, Attali J, Valensi P. Markers for silent myocardial ischemia in diabetes. Are they helpful? Diabetes \& metabolism. 2005;31(2):205-13.

22. Al-Humaidi G, Sarikaya I, Elgazzar AH, Owunwanne A. Myocardial perfusion abnormalities in asymptomatic type 2 diabetic patients. Journal of the Saudi Heart Association. 2018;30(1):3-8.

23. Mohagheghie A, Ahmadabadi M, Hedayat D, Pourbehi M, Assadi M. Myocardial perfusion imaging using technetium-99m sestamibi in asymptomatic diabetic patients. Nuklearmedizin. 2011;50(01):3-8.

24. Wannamethee SG, Shaper AG, Whincup PH, Lennon L, Sattar N. Impact of diabetes on cardiovascular disease risk and all-cause mortality in older men: influence of age at onset, diabetes duration, and established and novel risk factors. Archives of internal medicine. 2011;171(5):404-10.

25. Resl M, Vila G, Heinzl M, Luger A, Neuhold S, Prager R, et al. Cardiovascular risk prediction in diabetes-changes in the prognostic values of modern cardiovascular biomarkers in relation to duration of diabetes mellitus. 2020.

26. Fox CS, Sullivan L, D'Agostino RB, Wilson PW. The significant effect of diabetes duration on coronary heart disease mortality: the Framingham Heart Study. Diabetes care. 2004;27(3):704-8.

27. Wu Y-T, Chien C-L, Wang S-Y, Yang W-S, Wu Y-W. Gender differences in myocardial perfusion defect in asymptomatic postmenopausal women and men with and without diabetes mellitus. Journal of Women's Health. 2013;22(5):439-44. 
28. Salehi Y, Fard-Esfahani A, Fallahi B, Aghahosseini F, Beiki D, Emami-Ardekani A, et al. The myocardial perfusion scintigraphy in asymptomatic diabetic patients. Iranian Journal of Nuclear Medicine. 2015;23(1):27-35.

29. Malik D, Mittal B, Sood A, Parmar M, Kaur G, Bahl A. Left ventricular mechanical dyssynchrony assessment in long-standing type II diabetes mellitus patients with normal gated SPECT-MPI. Journal of Nuclear Cardiology. 2019;26(5):1650-8.
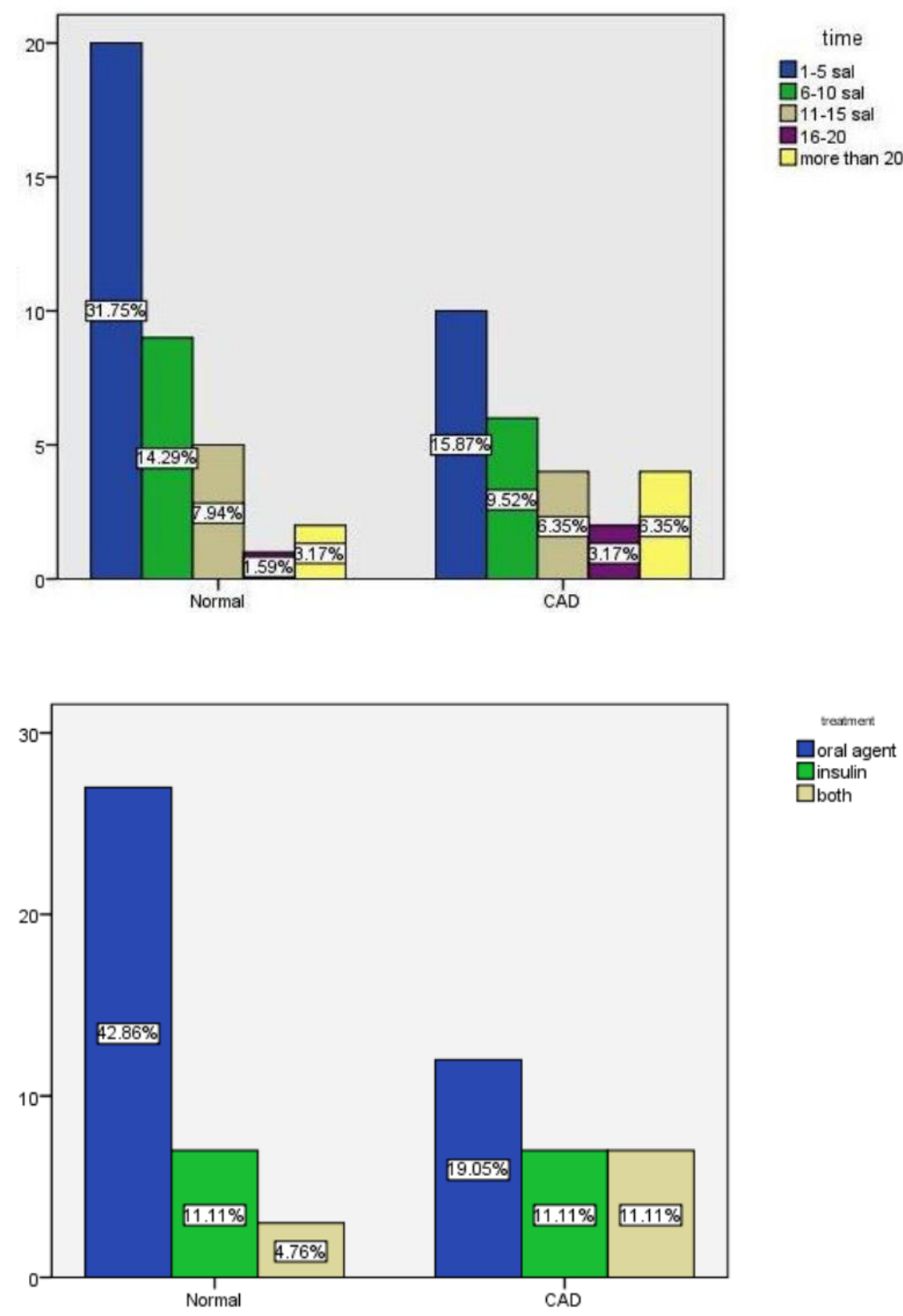

\section{Hosted file}


Table 1.pdf available at https://authorea.com/users/413280/articles/521667-assessing-theprevalence-and-predicting-factors-of-an-abnormal-gated-myocardial-perfusion-spect-inasymptomatic-patients-with-type-2-diabetes

\section{Hosted file}

Table 2.pdf available at https://authorea.com/users/413280/articles/521667-assessing-theprevalence-and-predicting-factors-of-an-abnormal-gated-myocardial-perfusion-spect-inasymptomatic-patients-with-type-2-diabetes

\section{Hosted file}

Table 3.pdf available at https://authorea.com/users/413280/articles/521667-assessing-theprevalence-and-predicting-factors-of-an-abnormal-gated-myocardial-perfusion-spect-inasymptomatic-patients-with-type-2-diabetes 